\title{
Predicting the development of stress urinary incontinence 3 years after hysterectomy
}

\author{
Mariëlle M. E. Lakeman • C. Huub Van Der Vaart • \\ Jan Willem Van Der Steeg • Jan-Paul W. R. Roovers • \\ On behalf of the HysVA study group
}

Received: 26 November 2010 / Accepted: 22 March 2011 / Published online: 12 April 2011

(C) The Author(s) 2011. This article is published with open access at Springerlink.com

\begin{abstract}
Introduction and hypothesis We aimed to develop a prediction rule to predict the individual risk to develop stress urinary incontinence (SUI) after hysterectomy.

Methods Prospective observational study with 3-year follow-up among women who underwent abdominal or vaginal hysterectomy for benign conditions, excluding vaginal prolapse, and who did not report SUI before surgery $(n=183)$. The presence of SUI was assessed using a validated questionnaire.

Results Significant prognostic factors for de novo SUI were BMI (OR 1.1 per $\mathrm{kg} / \mathrm{m}^{2}, 95 \%$ CI 1.0-1.2), younger age at time of hysterectomy (OR 0.9 per year, 95\% CI 0.8-1.0) and vaginal hysterectomy (OR 2.3, 95\% CI 1.0-5.2). Using these

HysVA study group: J.G. van der Bom, Leiden University Medical Centre; Y.A.J.M. Dabekausen, Streekziekenhuis Het Spittaal, Zutphen; M.J. Duk, Eemland Ziekenhuis, Amersfoort; E.J.M. van Erp, Leyenburg Ziekenhuis, Den Haag; J.F. ter Haar, TweeSteden Ziekenhuis, Tilburg, A.P.M. Heintz, University Medical Centre Utrecht; H.M.M.H. Kerkhof, Carolus Ziekenhuis, Den Bosch; W.F.A. Mensink, Martini Ziekenhuis, Groningen; L.C. van Otterlo Ziekenhuis Centrum, Apeldoorn; A.A.F. Planken, Mesos Medisch Centrum, Utrecht; J.P.W.R. Roovers, University Medical Centre Utrecht; J.H. Schagen van Leeuwen, Antonius Ziekenhuis, Nieuwegein; P.C.

Scholten, Diakonessenhuis, Utrecht; C.H. van der Vaart, University Medical Centre Utrecht, H.A.M. Vervest, St. Elisabeth Ziekenhuis, Tilburg; G.A. Wennink, Mesos Medisch Centrum Utrecht.
\end{abstract}

M. M. E. Lakeman $(\bowtie) \cdot J$. W. Van Der Steeg •

J.-P. W. R. Roovers

Department of Obstetrics and Gynaecology,

Academic Medical Centre,

Room H4-205, PO Box 22700, 1105 DE Amsterdam,

The Netherlands

e-mail: m.m.lakeman@amc.uva.nl

C. H. Van Der Vaart

Department of Obstetrics and Gynaecology,

University Medical Centre,

Utrecht, The Netherlands variables, we developed the following rule to predict the risk of developing SUI: $32+$ BMI - age $+(7.5 \times$ route of surgery $)$. Conclusions We defined a prediction rule that can be used to counsel patients about their individual risk on developing SUI following hysterectomy.

Keywords Hysterectomy · Prognosis · Stress urinary incontinence

\section{Introduction}

Hysterectomy is a frequently mentioned risk factor for the development of stress urinary incontinence (SUI) [1-3]. However, one could question if hysterectomy is really the cause of SUI or if women already suffered from SUI before surgery but failed to report this to their gynaecologist. Roovers et al. reported that $30 \%$ of the women before hysterectomy were already bothered by SUI compared to $8 \%$ in a random population sample [4]. All these women were not treated for SUI since they did not mention this to their treating physician [4]. Considering these data, it is not surprising that so many hysterectomized women undergo surgery for SUI [1].

However, the operation itself could also increase the risk by damaging anatomical structures involved in urethral support or the innervation of the urethral sphincter. This damage can be caused by direct surgical trauma or by trauma due to stretching or compression of the tissue [5]. During vaginal hysterectomy, the paravaginal tissue might be more dissected, thereby disrupting the pelvic neurons passing from the lateral aspect of the vagina and along the anterior wall [5, 6]. Since the pudendal nerve also travels close by the cervix, removal of the cervix might also result in loss of innervation [5]. The route of surgery might therefore play an important role in the risk of developing SUI [3]. 
Other previously described risk factors of the occurrence of SUI are high BMI, multiparity and increasing age [7-9]. Whether the documented increased risk of SUI after hysterectomy is mainly explained by differences in these risk factors or whether the surgical approach itself is an independent risk factor for de novo SUI is not well known.

Since hysterectomy is often performed to improve the quality of life, it is important to know what the risk of developing SUI is. Especially since this condition is known to negatively influence the quality of life. Therefore, our study is aimed to construct a prediction rule that adequately predicts the risk to develop SUI. If surgical approach proves to be an independent risk factor, a rule that includes this factor would be valuable to improve counselling and modify or abandon the procedure in patients that are at high risk to develop SUI.

\section{Material and methods}

Data for this study was collected as part of a prospective observational study in 13 teaching hospitals in the Netherlands [10]. All women who underwent hysterectomy for benign disease between January 1999 and July 2001 were included. We excluded patients undergoing hysterectomy for endometriosis or symptomatic prolapse as these conditions have been shown to affect pelvic floor function itself. Since we intended to calculate the risk of developing de novo SUI, we also excluded women who already reported SUI before hysterectomy. Written informed consent was obtained from all participating women. The medical ethic committee in all participating hospitals agreed with the study. Gynaecologists were free to choose a surgical route. If abdominal hysterectomy was performed, the cervix was removed unless the patient preferred to preserve the cervix or there were technical limitations to remove the cervix during surgery. All hysterectomies were performed according to a standardized protocol.

Peri-operative treatment was standardized [10, 11]. All patients were asked to complete the validated Dutch version of the urogenital distress inventory (UDI) at 2-4 weeks before surgery and at 6 weeks, 6 months, 1 year and 3 years after surgery [12, 13]. For this study, we used the questionnaire before surgery and 3 years after surgery.

The UDI consists of 19 items and each item measures if a micturition symptom is present and to what extend the patient is bothered by this symptom. The latter is measured on a four-point Likert scale ranging from not at all to severely bothered $[12,13]$. For this study, we used the question 'do you experience any urine leakage during exercise, sneezing or coughing' as index question for SUI.

We regarded a symptom to be bothersome if the latter was as 'I am bothered', 'I am much bothered' or 'I am severely bothered'. We regarded symptoms not to be bothersome when scored as 'not at all bothered' or when the symptom was not present. Women also received a questionnaire about their medical and obstetrical history.

\section{Statistical analysis}

The main goal of the analysis was to describe the importance of patient characteristics and operative variables on the risk of SUI after hysterectomy. Development of SUI 3 years after surgery was our primary endpoint. Variables of interest were BMI, age, parity, route of hysterectomy and removal or preservation of the cervix. We checked the linearity of the association between continuous variables BMI, age and parity versus the risk on SUI using visual inspection and spline functions. Univariable odds ratios $(\mathrm{OR})$, beta coefficients $(\beta)$ were calculated for all variables. Significance was determined by both $95 \%$ confidence intervals $(\mathrm{CI})$ and $P$ values.

\section{Development of prediction model}

Multivariable logistic regression analysis with a stepwise forward selection procedure was used to construct a prediction model for the risk of de novo bothersome SUI 3 years after hysterectomy. We selected variables with a $p$ value $<0.15$ for inclusion in the multivariable model since a more narrow inclusion could cause us to miss any important confounders [14].

\section{Calculation of the risk score}

The prediction model was converted into a prediction rule by using the regression coefficients. The risk score was expressed as the absolute value of the sum of the products of the variables and their $\beta$ coefficients from the final multivariable logistic regression model. The prediction rule was converted into a more easily applied diagnostic rule by dividing through the smallest regression coefficient and rounding it to the nearest integer. A constant was added to create outcome scores above 0 .

\section{Validation}

Internal validation was performed by using bootstrapping. This technique assesses the possible overfit of the created model. Bootstrapping is a technique to create comparable populations [15, 16]. For each group of 200 bootstrap samples, the model was refitted and tested against the observed sample in order to derive an estimate of the predictive accuracy and bias. We bootstrapped 200 times, in each of these new datasets the same multivariable logistic regression was used. 


\section{Accuracy of the model}

Calibration, the agreement between the predicted probabilities and the observed frequencies of having SUI, was tested with the Hosmer-Lemeshow test, where $p>0.05$ reflects good agreement. The discriminative ability was quantified using the area under the receiver operating characteristic curve (ROC curve) [15]. An area under the ROC curve of 0.5 implies that the diagnostic test does not exceed change, whereas an area of 1 implies that the predictive value of the test is perfect. As the ROC area reflects only the overall discriminative value of a model and not directly its clinical value. We additionally estimated the number of correctly and falsely predicted patients.

\section{Results}

In this prospective study, a total number of 234 patients were approached, of whom 183 women (78\%) responded at 3-year follow-up. No statistical significant differences were found in patient characteristics between women who did and did not complete the questionnaire (data not shown). Further analysis is restricted to the 183 patients who responded to the questionnaire 3 years after surgery.

The distribution of the different surgical techniques was similar in all participating centres. Sub analysis comparing surgical outcome data between the different participating centres also did not show that surgical outcome differed between participating centres. The operations in this study were performed by 57 different gynaecologists; none of the gynaecologists performed more than $8 \%$ of the procedures.

Table 1 shows the patient characteristics of all women without bothersome SUI before surgery. Bothersome SUI had developed in 40 women (22\%) 3 years after surgery. Analyses with spline functions demonstrated a linear association between the probability of developing bothersome SUI and continuous variables BMI, age and parity.

The results of univariable analysis are shown in Table 2. The results of the model formed using multivariable regression analysis are also shown in Table 2. Internal validation using bootstrapping indicated a possible overfit of the model up to $13 \%$. The calibration of the model was good (Hosmer-Lemeshow test $p=0.68$ ). The discriminative ability of the model as quantified using the area under the ROC curve was 0.69 (95\% CI 0.59-0.79).

A prediction rule was constructed using the regression coefficients $(\beta)$ of all variables which were selected in the multivariable model. After dividing all regression coefficients through 0.11 (the smallest regression coefficient), we constructed the following prediction rule: risk score $=32+\mathrm{BMI}-$ age $+(7.5 \times$ route of surgery $)$. Women undergoing vaginal hysterectomy received 1 point and women undergoing abdominal hysterectomy received 0 points at route of surgery. The score could not be calculated in three women because of missing data, one of these women developed bothersome SUI. Scores in our population ranged from 0 to 32 .

After calculating the score, the predicted risk of developing bothersome SUI can be found in Fig. 1. In the
Table 1 Patient characteristics of the included patients (i.e. patients without stress urinary incontinence who were scheduled for hysterectomy) $(N=183)$

\footnotetext{
${ }^{a}$ One patient can have more indications for hysterectomy
}

\begin{tabular}{lll}
\hline BMI $\left(\mathrm{kg} / \mathrm{m}^{2}\right)$ (mean, SD) & 24.8 & $(3.8)$ \\
Age (years) (mean, SD) & 43.6 & $(5.7)$ \\
Parity (median, range) & 2 & $(0-7)$ \\
Vaginal delivery & 0 & $(0-3)$ \\
Caesarean section & 9 & $(5-30)$ \\
Ultrasonographic size uterus in cm (median, range) & -5 & $(-10-0)$ \\
Uteral descent under anaesthesia in cm (median, range) & 63 & $(34.4)$ \\
History of abdominal surgery $(n, \%)$ & & $(65)$ \\
Indication for hysterectomy $(n, \%)^{\mathrm{a}}$ & 118 \\
Menorrhagia & 54 & $(30)$ \\
Metrorrhagia & 79 & $(43)$ \\
Abdominal pain & 44 & $(24)$ \\
Dysmenorrhea & & \\
Mode of surgery $(n, \%)$ & 42 & $(23)$ \\
Vaginal hysterectomy & 141 \\
Abdominal hysterectomy & 89 & $(63)$ \\
Total hysterectomy & & $(37)$ \\
Subtotal hysterectomy & 52 & $(6)$ \\
Concomitant salpingo-oophorectomy & 11 & \\
\hline
\end{tabular}


Table 2 Outcomes of univariable analysis and multivariable analysis using logistic regression with stepwise forward selection, of the association of patient characteristics and operative parameters, with the presence of post-operative bothersome stress incontinence 3 years after operation

\begin{tabular}{|c|c|c|c|c|c|c|c|c|}
\hline & \multicolumn{4}{|c|}{ Univariable analysis } & \multicolumn{4}{|c|}{ Multivariable analysis } \\
\hline & OR & $95 \% \mathrm{CI}$ & $P$ value & $\beta$ & OR & $95 \% \mathrm{CI}$ & $P$ value & $\beta$ \\
\hline BMI (per kg/m²) & 1.1 & $1.0-1.2$ & 0.09 & 0.08 & 1.1 & $1.0-1.2$ & 0.03 & 0.11 \\
\hline Age (per year) & 0.9 & $0.9-1.0$ & $<0.01$ & -0.09 & 0.9 & $0.8-1.0$ & $<0.01$ & -0.11 \\
\hline Vaginal delivery & 0.9 & $0.6-1.1$ & 0.30 & -0.16 & & & & \\
\hline Vaginal vs abdominal hysterectomy & 1.9 & $0.9-4.1$ & 0.11 & 0.64 & 2.3 & $1.0-5.2$ & 0.05 & 0.83 \\
\hline Total vs subtotal hysterectomy & 1.1 & $0.5-2.3$ & 0.89 & 0.06 & & & & \\
\hline Constant & & & & & 1.2 & & 0.90 & 0.22 \\
\hline
\end{tabular}

$B M I$ body mass index $\left(\mathrm{kg} / \mathrm{m}^{2}\right), O R$ odds ratio, $P \mathrm{p}$ value, $\beta$ regression coefficient, $C I$ confidence interval

case of a 40-year-old patient with a BMI of $25 \mathrm{~kg} / \mathrm{m}^{2}$ scheduled to undergo abdominal hysterectomy, the score would be: $32+25-40+(7.5 \times 0)=17$, when looking in Fig. 1, this means a $23 \%$ chance of developing bothersome SUI 3 years after hysterectomy. If the same patient is scheduled for vaginal hysterectomy, her score is: $32+25-$ $40+(7.5 \times 1)=24.5$, which means a $40 \%$ of developing bothersome SUI 3 years after surgery.

In Table 3, sensitivity and specificity are shown. For example, the sensitivity of the scoring rule to predict that women will develop bothersome SUI 3 years after surgery was $85 \%$ for a score above ten points ( 33 of the 39 women who developed bothersome SUI had a score $>10$ ). The specificity to predict that women will not develop bothersome SUI was $27 \%$ for women with a score below ten points (38 of the 141 women who did not develop bothersome SUI had a score below 10). The positive and negative predictive values are also shown in Table 3.

\section{Discussion}

In this study, we constructed a prediction rule to calculate the individual risk to develop bothersome SUI after hysterectomy. Identified risk factors were BMI, age and surgical route of hysterectomy. Using these parameters in the presented scoring rule enables the physician to calculate the risk for the individual patient on developing bothersome SUI 3 years after hysterectomy.

Before interpreting the data, some issues need to be addressed. We performed an observational study to compare the influence of different risk factors on SUI after
Fig. 1 Risk score chart, with $95 \%$ confidence interval of the prediction rule to estimate the chance of developing SUI 3 years after hysterectomy. Scores are calculated using the developed prediction rule: risk score $=32+$ BMI - age $+(7.5 \times$ route of surgery)

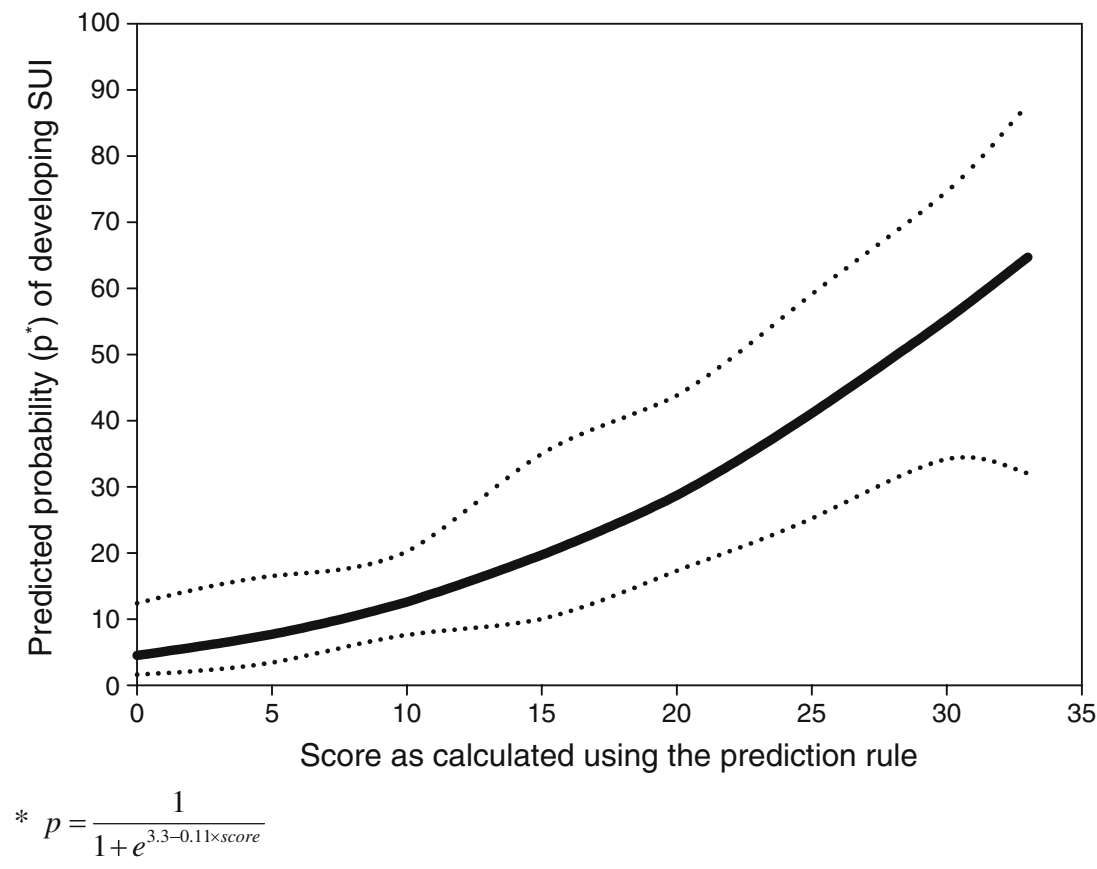


Table 3 Absolute number of patients, patients with stress urinary incontinence (SUI), sensitivity, specificity, positive and negative predictive value for predicting the development of SUI 3 years after hysterectomy according to the score as calculated using the presented scoring rule $($ score $=32+\mathrm{BMI}-\mathrm{age}+(7.5 \times$ route of surgery $))$

\begin{tabular}{lllcccc}
\hline & No. of patients & No. of patients with SUI & Sensitivity & Specificity & Positive predictive value & Negative predictive value \\
\hline Score $>5$ & 164 & 38 & 0.97 & 0.11 & 0.23 & 0.94 \\
Score $>10$ & 136 & 33 & 0.85 & 0.27 & 0.24 & 0.86 \\
Score $>15$ & 88 & 26 & 0.67 & 0.56 & 0.30 & 0.86 \\
Score $>20$ & 41 & 18 & 0.46 & 0.84 & 0.44 & 0.85 \\
Score $>25$ & 14 & 9 & 0.23 & 0.96 & 0.64 & 0.82 \\
\hline
\end{tabular}

SUI stress urinary incontinence

hysterectomy with a follow-up till 3 years post-operative. Because patients were not randomized, prognostic incomparability could have appeared. However, a randomized design would have limited the clinical application of the study results as in such RCT, only patients in whom both approaches are technically feasible, would have been included. As all prognostic variables were prospectively documented, we could correct for all factors which were expected to influence the risk of developing SUI in a multivariable regression analysis.

As a rule of thumb, for every ten observations, one variable can be included in the model. Since we have 40 observations, these means 4 variables, we included 5 variables, which means that our model is more at risk for being too optimistic. Therefore, we have estimated the size of overoptimism, the possible overfit was up to $13 \%$.

One of the concerns about this study may be that the observed effects are explained by differences in the gynaecologists' skills to perform surgery. However, by providing a detailed description of the surgical techniques in the study protocol, variation in the performance of surgery between gynaecologists has been limited. Sub analysis of our data did not show that surgical outcome differed between centres. As the surgical procedures were performed in 13 different hospitals by 57 different gynaecologists, we are confident about the generalizability of our results.

One might wonder why so few of the women with bothersome SUI received treatment for SUI. This is explained by the fact that this was a questionnaire study, the results of this questionnaire were not reported to the patients' gynaecologist, and based on the results, we did not approach patients for treatment.

The strength of this study is that the same validated questionnaires were used pre-operatively and postoperatively until 3 years post-operatively. The high response rate at 3-year follow-up shows that the questions could be consistently answered, but also represents the effort of the research team to keep all patients motivated to continue their participation in this study.
A scoring rule was calculated to assess the individual risk on de novo SUI. Internal validation was performed; however, external validation and impact analysis has to be performed to see if the rule is robust [17]. Variables in this scoring rule appeared to be BMI, age and surgical route.

The increased risk of SUI with increasing BMI has previously been described [7, 9, 18]. Cross-sectional studies showed that the increased risk of SUI with increasing BMI might best be explained through the mechanical impact of central adiposity on intra-abdominal pressure and urethral mobility [18]. The influence of BMI in this group is the same as it is in the normal population consisting of mainly non-hysterectomized women.

Higher age has also been described as an important risk factor for developing SUI in previous literature [7, 9, 18, 19]. The opposite was observed, however, in our study, suggesting that higher age at the time of hysterectomy protects against developing SUI. An explanation for the protective effect of higher age during hysterectomy might be that women, in whom ageing has not resulted in the development of SUI, are less susceptible for this symptom, even if they undergo a hysterectomy.

Our study revealed vaginal approach of hysterectomy also as independent risk factor for de novo SUI posthysterectomy. A possible explanation is that during vaginal hysterectomy, a larger amount of downwards traction is applied to the cervix and its surrounding tissue which can cause more supportive tissue damage. The same effect has been described after vaginal delivery compared to caesarean section [20, 21]. In a similar way, the continuous downwards traction might damage the pudendal nerve and contribute to irreversible damage of the pelvic innervation.

Another explanation for the increased risk of developing SUI after vaginal hysterectomy might be the fact that women scheduled for vaginal hysterectomy had probably more uterine descent caused by multiple vaginal deliveries. Therefore, we also included the number of vaginal deliveries in the model. However, the number of vaginal deliveries in the past did not seem to influence the risk of developing SUI in our population. 
Using these few and simply obtainable variables in the presented scoring rule enables the physician to calculate the risk that a woman will develop SUI. This could be a valuable tool in counselling women before hysterectomy. Of course this increased risk of developing SUI should be balanced against the increased risk of procedure-related complications, increased duration of hospitalisation and increased health care costs associated with abdominal hysterectomy [22]. But women with a high probability could be counselled about this risk, and more postoperative care, for example pelvic floor physiotherapy, can be taken for this specific group.

In conclusion, we are the first to develop a prediction rule which predicts the development of bothersome SUI after hysterectomy. The developed rule still has to be externally validated and impact analysis has to be performed. However, the developed prediction rule is able to identify the individual risk and can hereby improve the quality of counselling about the risk of SUI after hysterectomy.

Details of ethics approval The medical ethical committee of the University Medical Centre Utrecht approved of the study before the start of the study in 1999. The study was also approved by all local ethical committees.

Funding No funding was received for this publication.

\section{Conflicts of interest None.}

Open Access This article is distributed under the terms of the Creative Commons Attribution Noncommercial License which permits any noncommercial use, distribution, and reproduction in any medium, provided the original author(s) and source are credited.

\section{References}

1. Altman D, Granath F, Cnattingius S, Falconer C (2007) Hysterectomy and risk of stress-urinary-incontinence surgery: nationwide cohort study. Lancet 370:1494-1499

2. Brown JS, Sawaya G, Thom DH, Grady D (2000) Hysterectomy and urinary incontinence: a systematic review. Lancet 356:535-539

3. Lakeman MM, van der Vaart CH, Roovers JP (2010) Hysterectomy and lower urinary tract symptoms: a nonrandomized comparison of vaginal and abdominal hysterectomy. Gynecol Obstet Investig 70:100-106

4. Roovers JP, van der Vaart CH, van der Bom JG, Heintz AP (2000) Urinary incontinence after hysterectomy. Lancet 356:2012-2013
5. Parys BT, Haylen BT, Hutton JL, Parsons KF (1989) The effects of simple hysterectomy on vesicourethral function. Br J Urol 64:594-599

6. Smith PH, Ballantyne B (1968) The neuroanatomical basis for denervation of the urinary bladder following major pelvic surgery. Br J Surg 55:929-933

7. Hampel C, Artibani W, Espuna PM, Haab F, Jackson S, Romero J, Gavart S, Papanicolaou S (2004) Understanding the burden of stress urinary incontinence in Europe: a qualitative review of the literature. Eur Urol 46:15-27

8. Hannestad YS, Rortveit G, Sandvik H, Hunskaar S (2000) A community-based epidemiological survey of female urinary incontinence: the Norwegian EPINCONT study. Epidemiology of incontinence in the county of nord-trondelag. J Clin Epidemiol 53:1150-1157

9. Minassian VA, Stewart WF, Wood GC (2008) Urinary incontinence in women: variation in prevalence estimates and risk factors. Obstet Gynecol 111:324-331

10. Roovers JP, van der Bom JG, van der Vaart CH, Fousert DM, Heintz AP (2001) Does mode of hysterectomy influence micturition and defecation? Acta Obstet Gynecol Scand 80:945-951

11. Roovers JP, van der Bom JG, van der Vaart CH, Heintz AP (2003) Hysterectomy and sexual wellbeing: prospective observational study of vaginal hysterectomy, subtotal abdominal hysterectomy, and total abdominal hysterectomy. BMJ 327:774-778

12. Shumaker SA, Wyman JF, Uebersax JS, McClish D, Fantl JA (1994) Health-related quality of life measures for women with urinary incontinence: the Incontinence Impact Questionnaire and the Urogenital Distress Inventory. Continence Program in Women (CPW) Research Group 2. Qual Life Res 3:291-306

13. van der Vaart CH, de Leeuw JR, Roovers JP, Heintz AP (2003) Measuring health-related quality of life in women with urogenital dysfunction: the urogenital distress inventory and incontinence impact questionnaire revisited. Neurourol Urodyn 22:97-104

14. Twisk JWR (2007) Inleiding in de toegepaste biostatistiek. Elsevier Gezondheidszorg, Maarssen

15. Steyerberg EW (2009) CLinical prediction models. Springer

16. Steyerberg EW, Bleeker SE, Moll HA, Grobbee DE, Moons KG (2003) Internal and external validation of predictive models: a simulation study of bias and precision in small samples. J Clin Epidemiol 56:441-447

17. Leushuis E, van der Steeg JW, Steures P, Bossuyt PM, Eijkemans MJ, van der Veen F, Mol BW, Hompes PG (2009) Prediction models in reproductive medicine: a critical appraisal 1. Hum Reprod Update 15:537-552

18. Parazzini F, Chiaffarino F, Lavezzari M, Giambanco V (2003) Risk factors for stress, urge or mixed urinary incontinence in Italy. BJOG 110:927-933

19. Thom DH, Van den Eeden SK, Brown JS (1997) Evaluation of parturition and other reproductive variables as risk factors for urinary incontinence in later life. Obstet Gynecol 90:983-989

20. Dietz HP, Clarke B, Vancaillie TG (2002) Vaginal childbirth and bladder neck mobility. Aust N Z J Obstet Gynaecol 42:522-525

21. Toozs-Hobson P, Balmforth J, Cardozo L, Khullar V, Athanasiou $S$ (2008) The effect of mode of delivery on pelvic floor functional anatomy. Int Urogynecol J Pelvic Floor Dysfunct 19:407-416

22. Warren L, Ladapo JA, Borah BJ, Gunnarsson CL (2009) Open abdominal versus laparoscopic and vaginal hysterectomy: analysis of a large United States payer measuring quality and cost of care. J Minim Invasive Gynecol 16:581-588 